\title{
Neuropsychological function after endovascular and neurosurgical treatment of subarachnoid hemorrhage: a systematic review and meta-analysis
}

\author{
Peter Egeto, MA, ${ }^{1}$ R. Loch Macdonald, MD, PhD, ${ }^{2-4,6}$ Tisha J. Ornstein, PhD, CPsych, ${ }^{1}$ and \\ Tom A. Schweizer, PhD2-6 \\ 'Department of Psychology, Ryerson University; ${ }^{2}$ Keenan Research Centre for Biomedical Science and ${ }^{3}$ Division of \\ Neurosurgery, St. Michael's Hospital; and Institutes of ${ }^{4}$ Medical Science and 5 Biomaterials and Biomedical Engineering and \\ ${ }^{6}$ Department of Neurosurgery, Faculty of Medicine, University of Toronto, Ontario, Canada
}

\begin{abstract}
OBJECTIVE Subarachnoid hemorrhage (SAH) is treated with either surgical clipping or endovascular coiling, though the latter is the preferred treatment method given its more favorable functional outcomes. However, neuropsychological functioning after treatment is rarely taken into account. In this meta-analysis, the authors synthesized relevant data from the literature and compared neuropsychological functioning in patients after coiling and clipping of SAH. They hypothesized that the coiled patients would outperform the clipped patients; that group differences would be greater with higher posterior circulation rupture rates, in older patients, and in more recent publications; that group differences would be smaller with greater rates of middle cerebral artery (MCA) rupture; and that anterior communicating artery (ACoA) rupture rates would not influence effect sizes.
\end{abstract}

METHODS The MEDLINE, Embase, and PsycINFO databases were searched for clinical studies that compared neuropsychological functioning after either endovascular coiling or surgical clipping for $\mathrm{SAH}$. Hedge's g and 95\% confidence intervals were calculated using random effects models. Patients who had undergone coiling or clipping were compared on test performance in 8 neuropsychological domains: executive functions, language, attention/processing speed, verbal memory, visual memory, spatial memory, visuospatial functions, and intelligence. Patients were also compared with healthy controls, and meta-regressions were used to explore the relation between effect sizes and publication year, delay between treatment and neuropsychological testing, mean patient age, and rates of posterior circulation, ACoA, and MCA ruptures.

RESULTS Thirteen studies with 396 clipped cases, 314 coiled cases, and 169 healthy controls were included in the study. The coil-treated patients outperformed the clip-treated patients on executive function $(\mathrm{g}=0.17,95 \% \mathrm{Cl} 0.08-0.25)$ and language tests $(\mathrm{g}=0.23,95 \% \mathrm{Cl} 0.07-0.39)$, and all patients were impaired relative to healthy controls ( $\mathrm{g}$ ranged from -0.93 to -0.29 ). Coiled patients outperformed clipped patients to a greater degree in more recent publications, over longer posttreatment testing delays, and among older patients. Higher rates of posterior circulation and MCA aneurysms were associated with smaller group differences, while ACoA rupture rates did not influence effect sizes.

CONCLUSIONS Coiling of SAH may promote superior neuropsychological functioning under certain circumstances and could have applications for the specialized care of SAH patients.

https://thejns.org/doi/abs/10.3171/2016.11.JNS162055

KEY WORDS subarachnoid hemorrhage; endovascular coiling; surgical clipping; neuropsychology; vascular disorders

$\mathrm{S}$ PONTANEOUS subarachnoid hemorrhage ( $\mathrm{SAH})$ is caused primarily by the rupture of aneurysms on cerebral arteries, allowing the entry of blood into the subarachnoid space. Although it accounts for only 5\% of all strokes, it has a mortality rate of $35 \% .{ }^{10}$ Importantly,
SAH affects younger individuals relative to ischemic stroke (mean 52 vs 70-80 years of age), contributing to high societal costs. ${ }^{28}$ Patients who survive are often burdened by neurological, cognitive, and functional impairment. ${ }^{2}$

Historically, surgical clipping was the most frequent

ABBREVIATIONS ACOA = anterior communicating artery; $\mathrm{HH}=$ Hunt and Hess; $\mathrm{MCA}=$ middle cerebral artery; SAH = subarachnoid hemorrhage; SCWIT = Stroop ColorWord Interference Test; TMT = Trail Making Test; WCST = Wisconsin Card Sorting Test; WFNS = World Federation of Neurosurgical Societies.

SUBMITTED August 5, 2016. ACCEPTED November 17, 2016.

INCLUDE WHEN CITING Published online April 14, 2017; DOI: 10.3171/2016.11.JNS162055. 
treatment for ruptured aneurysms, although endovascular coiling is becoming more common since its introduction in the 1990s. ${ }^{19}$ This is based largely on the International Subarachnoid Aneurysm Trial, ${ }^{21}$ which revealed that those treated via the endovascular approach have a somewhat lower risk of death and dependency at 1 year versus 2 months posttreatment (a relative risk reduction of 24\%).

A caveat to the treatment outcome data is that neuropsychological evaluation has not usually been a primary end point in clinical trials. An important question is whether cognitive outcomes differ after the 2 treatment approaches. In an attempt to answer this question, we performed a meta-analysis of published studies that evaluated the neuropsychological test performance of SAH patients after surgical clipping or endovascular coiling. Test performance was also compared with that of healthy controls where applicable, and the relationships between effect sizes and publication year, the delay between treatment and neuropsychological testing, mean patient age, and ruptured aneurysm location were modeled using meta-regressions.

\section{Methods \\ Literature Search}

We searched the MEDLINE, Embase, and PsycINFO databases for studies that compared neuropsychological functioning after either endovascular coiling or surgical clipping for SAH. The keywords, along with appropriate medical subject heading (MeSH) terms, used were "subarachnoid hemorrhage," "endovascular coil," "coil," "surgical clip," "aneurysm clip," "clip," "neuropsychological test," "neuropsychological assessment," and "cognitive." The search was restricted to clinical studies published in the English language up to March 3, 2016, performed in adults, and including separate neuropsychological test data for both coiled and clipped patients. Reference lists in the included studies were searched, and the authors of inaccessible papers were contacted in an attempt to obtain them. Case studies, reviews, and conference abstracts were excluded. The methods and findings of this systematic review followed Preferred Reporting Items for Systematic Reviews and Meta-Analyses (PRISMA) guidelines. ${ }^{20}$

\section{Data Synthesis and Analyses}

The meta-analyses and meta-regressions were performed using Comprehensive Meta-Analysis (version 2.0). ${ }^{4}$ Sample sizes, means, and standard deviations of test scores and demographic and clinical variables were extracted from the included studies. Hedge's g and 95\% confidence intervals were calculated using random effects models. Significance was set at $p=0.05$. Neuropsychological tests were grouped into 8 domains: executive functions (Digit Span backward and forward/backward combination, semantic fluency [total words at 15,45 , and 60 seconds, errors, total animals, and total fruits], phonemic fluency [total words at 15, 45, and 60 seconds and errors], verbal [combined] fluency, alternating fluency, verbal fluency intrusions, verbal fluency perseverative errors, Temporal Judgement test, Key Search Test, Five-Point Test correct and incorrect, Alternating Drawing Test, Color Trails Test trial 2, Iowa Gambling Task CD-AB score,
Letter Number Sequencing, Arithmetic, Wisconsin Card Sorting Test [WCST] category total, WCST perseverative and nonperseverative errors, Elithorn's Perceptual Maze Test, Modified Card Sorting Task category totals and errors, Stroop Color-Word Interference Test [SCWIT; total correct, errors, and interference score], Trail Making Test [TMT]-B time and errors, TMT B-A, TMT B/A, Digit Ordering Test, Intra-/Extra-Dimensional Set-Shift Task extradimensional attempts and stages complete, Spatial Working Memory strategy and between-set errors, Tower of London total correct, Brixton Test total and errors, Hayling Test, Working Memory Index, and Frontal Assessment Battery total), language (Graded Naming Test, spelling, sentence construction, Boston Naming Test, Montreal-Toulouse Protocol: Aphasia Test [comprehension, repetition, denomination, written comprehension, and reading]), attention/processing speed (TMT-A, digit copy, number cancellation, Symbol Digit Modalities Test, test of sustained attention [reaction time, misses, and false alarms], Go/No-Go [reaction time, misses, and false alarms], visual search, SCWIT color and word scores, Test of Everyday Attention [sustained, divided, and selective attention], and Processing Speed Index), visuospatial functions (silhouettes, cube test, form discrimination correct and incorrect, Perceptual Organization Index, and Visual Object and Space Perception Battery), verbal memory (word repetition, verbal learning, Logical Memory immediate and delayed, Recognition Memory Test words, Paired-Associate Learning Test immediate and delayed, word recognition, story recall immediate and delayed, $\mathrm{Au}-$ ditory Verbal Learning Test trials 1-5 and delayed, and Test de Aprendizaje Verbal España-Complutense [immediate, delayed, and total recall]), visual memory (Recognition Memory Test faces, Rey-Osterrieth Complex Figure Test [immediate, delayed, and recognition], figure recall, face recognition, visual immediate and delayed recall, and Doors Test), spatial memory (Corsi block-tapping task), and intelligence (Verbal IQ, Performance IQ, Full Scale IQ, Raven's Advanced Progressive Matrices, verbal judgment, matrices, vocabulary, and National Adult Reading Test). Recognition, immediate recall, and delayed recall scores were grouped together in the memory domains because of an insufficient sample size to separate them. Separate analyses were performed for the 2 studies that conducted follow-up testing; ;2,16 Frazer et al. retested patients 6 months after treatment, and Koivisto et al. retested at 3 and 12 months posttreatment. The relations between effect sizes and publication year, delay between treatment and neuropsychological testing, mean age of patients, and rates of posterior circulation, anterior communicating artery $(\mathrm{ACo} A)$, and middle cerebral artery $(\mathrm{MCA})$ ruptures were explored using linear meta-regression models. Study quality was assessed using the Newcastle-Ottawa Scale. ${ }^{31}$ Publication bias was assessed using Egger's regression test, ${ }^{8}$ Duval and Tweedie's trim and fill, ${ }^{7}$ and visual inspection of the funnel plot.

\section{Hypotheses}

Given the literature, we hypothesized that coiled patients would, for the most part, outperform clipped patients on neuropsychological tests and that all patients 
would perform worse than healthy controls. Given the mixed trend for greater improvements in coiling versus clipping efficacy over the last decade and a half, we hypothesized that publication year would modestly influence effect sizes (larger group differences in more recent studies $^{5}$ ). Provided some evidence for more efficacious outcomes after coiling versus clipping in older patients, we expected age to be related to greater effect sizes. ${ }^{5}$ Lastly, because of the purported influence of rupture location on treatment outcome, we examined the influence of posterior circulation, $\mathrm{ACoA}$, and MCA ruptures on effect sizes. Considering the somewhat superior outcomes for coiled versus clipped posterior circulation ruptures,,${ }^{14}$ we expected higher rates of posterior circulation ruptures to be associated with larger effect sizes. Conversely, because of the relative difficulty in coiling MCA aneurysms, ${ }^{24}$ we predicted that higher rates of MCA rupture would be associated with smaller effect sizes. Because ACoA ruptures were ubiquitous in the included studies, the effect of treatment modality was examined. However, given the lack of angioanatomical contraindications for either treatment, $\mathrm{ACOA}$ rupture rates were not predicted to influence effect sizes.

\section{Results \\ Literature Search}

The results of the literature search are depicted in Fig. 1. The initial search yielded 108 articles, from which 27 duplicates were removed. Of the 81 abstracts reviewed, 62 were excluded for failing to meet our inclusion criteria. After fully reviewing the remaining 19 studies, 6 were excluded for not reporting treatment group comparisons of neuropsychological tests, ${ }^{26,27,32}$ not including both clipped and coiled patients, ${ }^{1}$ or only providing pretreatment neuropsychological test data. ${ }^{30}$ Thirteen studies were included in our systematic review, all cohort desig ns. ${ }^{3,6,9,11-13,16-18,22,23,25,29}$ The mean study quality score (Newcastle-Ottawa Scale) was 5, but because of the variability in study designs there was no common total score (totals were either 7 or 8 ); thus, comparison between studies was not possible. The individual scores are listed in Table 1.

\section{Sample Characteristics}

The meta-analysis included 396 clipped patients, 314 coiled patients, and 169 healthy controls (Tables 1 and 2). The mean age among the clipped patients was $49.3 \pm$ $10.0,49.7 \pm 11.0$ among the coiled patients, and $52.6 \pm$ 10.9 among the healthy controls. Most of the patients had good clinical grades (for example, World Federation of Neurosurgical Societies [WFNS] Grades I-II). Aneurysm ruptures were distributed across the cerebrovascular system, though the majority were located in the anterior circulation, and some studies only reported on stroke patients with ACoA ruptures. ${ }^{6,9,11,23}$

\section{Coiled Versus Clipped Patients}

Patients treated with coiling outperformed those treated with clipping on measures of executive function (Hedge's $\mathrm{g}=0.17,95 \%$ CI $0.08-0.25)$ and language $(\mathrm{g}=0.23,95 \%$ CI 0.07-0.39), both small effects (Fig. 2). No significant

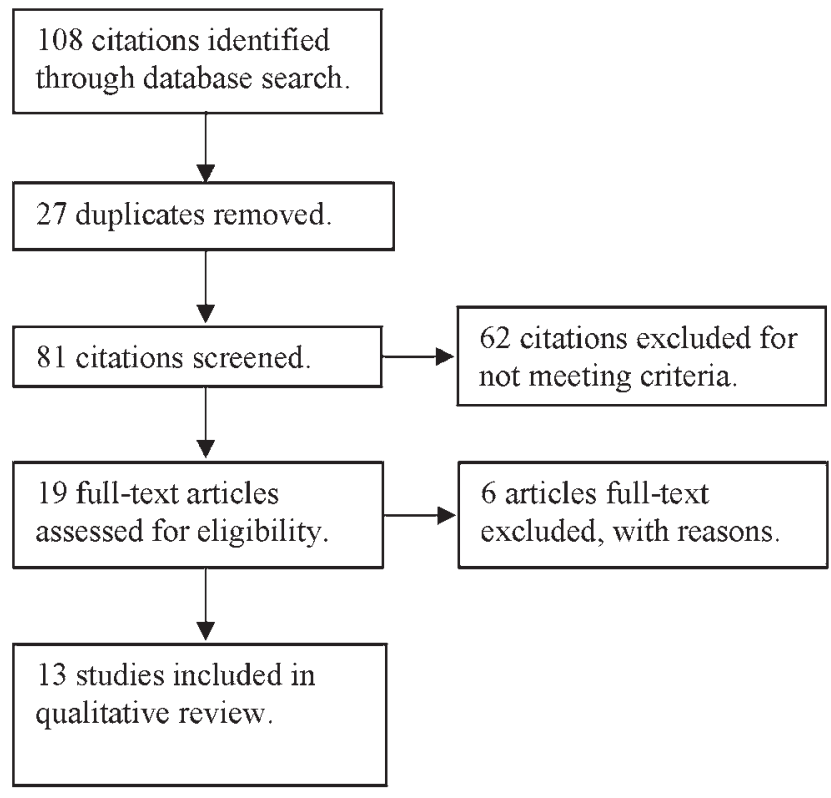

FIG. 1. Flowchart of the study selection process.

differences were found in the domains of attention/processing speed ( $g=0.09,95 \%$ CI -0.06 to 0.24$)$, intelligence $(\mathrm{g}=-0.03,95 \% \mathrm{CI}-0.31$ to 0.25$)$, spatial memory ( $\mathrm{g}=-0.07,95 \% \mathrm{CI}-0.42$ to 0.29 ), verbal memory ( $\mathrm{g}=$ $0.03,95 \%$ CI -0.12 to 0.17 ), visual memory ( $\mathrm{g}=0.02,95 \%$ CI -0.22 to 0.27$]$ ), or visuospatial functions ( $\mathrm{g}=-0.18$, $95 \%$ CI -0.39 to 0.03 ). No significant indices of publication bias were indicated by Egger's regression ( $\mathrm{Y}=0.11$, $95 \%$ CI -0.75 to $0.97, p>0.05$ ), Duval and Tweedie's trim and fill (no studies were trimmed), or visual inspection of the funnel plot.

\section{Analysis of Follow-Up Testing}

Two studies compared patients at follow-up sessions. ${ }^{12,16}$ Frazer et al. tested patients 6 months posttreatment, and Koivisto et al. tested patients at 3 and 12 months after treatment. In an analysis of the medium follow-up sessions (the 6- and 3-month intervals in Frazer et al. and Koivisto et al., respectively), clipped patients scored higher than the coiled patients on tests of executive function ( $\mathrm{g}=-0.22,95 \% \mathrm{CI}-0.43$ to -0.01$)$ and visuospatial functions ( $\mathrm{g}=-0.66,95 \% \mathrm{CI}-1.24$ to -0.07 ), small and medium effects, respectively. Clipped patients almost significantly outperformed the coiled patients on intelligence measures ( $\mathrm{g}=-0.52,95 \% \mathrm{CI}-1.07$ to 0.02$)$. No significant differences emerged on tests of attention/processing speed $(\mathrm{g}=-0.06,95 \% \mathrm{CI}-0.29$ to 0.17$)$, language ( $\mathrm{g}=$ $-0.19,95 \%$ CI -0.67 to 0.29 ), verbal memory ( $\mathrm{g}=0.11$, $95 \% \mathrm{CI}-0.17$ to 0.39 ), or visual memory ( $\mathrm{g}=-0.08,95 \%$ CI -0.39 to 0.24 ). No significant performance differences were maintained at the 12-month follow-up in the Koivisto study. It is noteworthy that the superior performance of clipped patients on almost all tests was unique to Frazer et al.; although other studies have found some differences favoring clipped patients, those in Frazer et al. performed better on almost all fronts, significantly or not. Since only 2 studies were included in the medium follow-up analysis, 
TABLE 1. Summary of patient demographics among studies of coiling versus clipping for SAH

\begin{tabular}{|c|c|c|c|c|c|c|c|}
\hline \multirow[b]{2}{*}{ Authors \& Year } & \multicolumn{3}{|c|}{ Sample Size } & \multicolumn{3}{|c|}{ Mean Age (SD) } & \multirow{2}{*}{$\begin{array}{c}\text { NOS Ratings } \\
\text { (score/ } \\
\text { possible total) }\end{array}$} \\
\hline & Clipped & Coiled & Controls & Clipped & Coiled & Controls & \\
\hline Bellebaum et al., 2004 & 16 & 16 & 16 & $54.37(12.54)$ & $54.0(12.91)$ & $54.19(13.01)$ & $7 / 8$ \\
\hline Chan et al., 2002 & 9 & 9 & 19 & $57.0(6.68)$ & $52.13(11.47)$ & $51.63(8.47)$ & $7 / 8$ \\
\hline Escartin et al., 2012 & 20 & 20 & 31 & $53.0(8.8)$ & $46.2(10.2)$ & $50(11.1)$ & $5 / 8$ \\
\hline Fontanella et al., 2003 & 20 & 17 & 18 & 52.6 (NA) & 54.14 (NA) & 53.4 (NA) & $2 / 8$ \\
\hline Frazer et al., 2007 & 12 & 11 & NA & $47.5(11.5)$ & $44.7(9.4)$ & NA & $3 / 7$ \\
\hline $\begin{array}{l}\text { Hadjivassiliou et al., } \\
2001\end{array}$ & 40 & 40 & 31 & 49.8 (NA) & 51.9 (NA) & NA & $5 / 8$ \\
\hline Koivisto et al., $2000^{*}$ & $\begin{array}{l}\text { 1st: 20, 2nd: } \\
\text { 30, 3rd: } 37\end{array}$ & $\begin{array}{l}\text { 1st: 25, 2nd: } \\
\text { 33, 3rd: } 38\end{array}$ & NA & $\begin{array}{c}\text { 1st: } 45.5 \text { (14.2), 2nd: } 48.1 \\
\text { (14.1), 3rd: } 49.2 \text { (13.2) }\end{array}$ & $\begin{array}{c}\text { 1st: } 45.4 \text { (13.7), 2nd: } 45.1 \\
\text { (14.2), 3rd: } 46.8 \text { (14.3) }\end{array}$ & NA & $5 / 7$ \\
\hline Ladowski et al., 2014 & 17 & 55 & 25 & $55.25(11.01)$ & $54.13(8.8)$ & $56.56(13.42)$ & $6 / 8$ \\
\hline Latimer et al., 2013 & 9 & 14 & NA & $53.3(12.7)$ & $52.2(5.7)$ & NA & $5 / 7$ \\
\hline Preiss et al., 2007 & 35 & 40 & NA & $44.4(12.1)$ & $46.9(10.7)$ & NA & $4 / 7$ \\
\hline Proust et al., 2009 & 36 & 14 & NA & $45.4(13.1)$ & $50.1(16.3)$ & NA & $7 / 7$ \\
\hline $\begin{array}{l}\text { Santiago-Ramajo et } \\
\text { al., } 2007\end{array}$ & 40 & 24 & 29 & $47.33(12.51)$ & $51.25(14.3)$ & $51.17(11.59)$ & $6 / 8$ \\
\hline Vieira et al., 2012 & 122 & 29 & NA & $50.05(1.62) \dagger$ & $50.05(1.62) \dagger$ & NA & $3 / 7$ \\
\hline
\end{tabular}

NA = not applicable; NOS = Newcastle-Ottawa Scale.

* Three testing sessions with increasingly larger samples and consequently different sample characteristics were included in this study: first session at discharge, second session 3 months posttreatment, and third session at 12 months posttreatment.

$\dagger$ Only the combined age of clipped and coiled patients was provided in this study.

the discordant results are probably attributable to the influence of the Frazer study.

\section{Coiled and Clipped Patients Versus Controls}

Patients treated with either treatment modality fared almost universally worse than the healthy controls (Figs. 3 and 4). Specifically, a worse performance emerged on measures of attention/processing speed (coiled: $\mathrm{g}=-0.29$, $95 \%$ CI -0.53 to -0.05 ; clipped: $\mathrm{g}=-0.60,95 \% \mathrm{CI}-0.87$ to -0.33 ), executive function (coiled: $\mathrm{g}=-0.49,95 \% \mathrm{CI}$ -0.61 to -0.38 ; clipped: $\mathrm{g}=-0.74,95 \% \mathrm{CI}-0.86$ to -0.62 ), intelligence (coiled: $\mathrm{g}=-0.43,95 \% \mathrm{CI}-0.81$ to -0.04 ; clipped: $\mathrm{g}=-0.93,95 \% \mathrm{CI}-1.33$ to -0.53 ), language (coiled: $\mathrm{g}=-0.40,95 \% \mathrm{CI}-0.71$ to -0.09 ; clipped: $\mathrm{g}=$ $-0.52,95 \%$ CI -0.92 to -0.12 ), verbal memory (coiled: $\mathrm{g}=-0.55,95 \%$ CI -0.88 to -0.23 ; clipped: $\mathrm{g}=-0.53$, 95\% CI -0.92 to -0.14 ), and visual memory (coiled: $\mathrm{g}=$ $-0.81,95 \%$ CI -1.15 to -0.46 ; clipped: $\mathrm{g}=-0.85,95 \% \mathrm{CI}$ -1.20 to -0.51$)$. No differences emerged on spatial memory (coiled: $\mathrm{g}=-0.34,95 \% \mathrm{CI}-0.72$ to 0.04 ; clipped: $\mathrm{g}$ $=-0.23,95 \% \mathrm{CI}-0.61$ to 0.14 ) or visuospatial functions (coiled: $\mathrm{g}=0.02,95 \% \mathrm{CI}-0.84$ to 0.88 ; clipped: $\mathrm{g}=-0.05$, $95 \% \mathrm{CI}-0.87$ to 0.76$)$. In general, the effects were medium and, in some cases, large, with greater deficits observed for clipped patients.

\section{Publication, Study Design, Demographic, and Clinical Variables}

The publication year, delay between treatment and neuropsychological testing, mean patient age, and rupture location data were entered into meta-regressions to model their relationships to test performance outcomes. Publi- cation year was found to be a significant predictor of effect sizes $(\beta=0.015,95 \%$ CI $0.0017-0.028, \mathrm{p}<0.05)$, accounting for $9 \%$ of the variance. Specifically, effect sizes (that is, performance differences) were somewhat greater in later versus earlier publications. Similarly, the testing delay also significantly predicted effect sizes $(\beta=0.0059$, $95 \%$ CI $0.0007-0.011, \mathrm{p}<0.05, \mathrm{R}^{2}=0.04$ ), which modestly increased with greater test delays. Mean patient age emerged as one of the greatest predictors $(\beta=0.049,95 \%$ CI 0.032-0.066, $\mathrm{p}<0.05$ ), accounting for $48 \%$ of the variance. Evidently, test score differences between treatment groups were greater when the mean patient age was older. In terms of rupture location, higher rates of posterior circulation ruptures predicted smaller effect sizes across all domains $(\beta=-2.23,95 \%$ CI -3.61 to $0.86, p<0.05$, $\mathrm{R}^{2}=0.25$ ). When broken down by neuropsychological domains, greater posterior circulation rupture rates significantly predicted lower intelligence scores $(\beta=-5.66$, $95 \% \mathrm{CI}-10.94$ to $0.38, \mathrm{p}<0.05, \mathrm{R}^{2}=0.36$ ); greater MCA rupture rates predicted greater effect sizes on intelligence measures $\left(\beta=3.37,95 \%\right.$ CI $\left.1.65-5.10, p<0.05, \mathrm{R}^{2}=0.74\right)$ but smaller effect sizes on language $(\beta=-1.99,95 \% \mathrm{CI}$ -3.48 to $\left.-0.50, \mathrm{p}<0.05, \mathrm{R}^{2}=1.00\right)$ and executive function tests $(\beta=-0.75,95 \% \mathrm{CI}-1.49$ to -0.022$], \mathrm{p}<0.05, \mathrm{R}^{2}=$ 0.18 ). Anterior communicating artery rupture rates did not predict effect size magnitude in any domain.

\section{Discussion}

Endovascular coiling has become a common method for repairing ruptured aneurysms..$^{19}$ Functional outcomes after coiling are often reported to be more favorable than those after the surgical approach, depending on aneurysm 
TABLE 2. Summary of characteristics of studies on coiling versus clipping for SAH

\begin{tabular}{|c|c|c|c|c|c|c|}
\hline \multirow[b]{2}{*}{ Authors \& Year } & \multicolumn{2}{|c|}{ Rupture Location } & \multicolumn{2}{|c|}{ Good Clinical Grade (\% patients) } & \multicolumn{2}{|c|}{ Mean Testing Delay in Mos (SD) } \\
\hline & Clipped & Coiled & Clipped & Coiled & Clipped & Coiled \\
\hline $\begin{array}{l}\text { Bellebaum et al., } \\
2004\end{array}$ & ACoA, ICA, MCA & $\begin{array}{c}\text { ACoA, ICA, MCA, } \\
\text { PCoA, PICA }\end{array}$ & $\mathrm{HH}: 81 \%$ & $\mathrm{HH}: 81 \%$ & $28.4(11.2)$ & $22.7(14.6)$ \\
\hline Chan et al., 2002 & $\mathrm{ACoA}$ & $\mathrm{ACoA}$ & $\mathrm{HH}: 2.0(0) \dagger$ & $\mathrm{HH}: 2.3(0.5) \dagger$ & $>12.0$ & $>12.0$ \\
\hline Escartin et al., 2012 & $\mathrm{ACOA}$ & $\mathrm{ACOA}$ & WFNS: $80 \%$ & WFNS: $89.5 \%$ & $36.6(20.7)$ & $32.5(26.5)$ \\
\hline $\begin{array}{l}\text { Fontanella et al., } \\
2003\end{array}$ & $\mathrm{ACoA}$ & $\mathrm{ACoA}$ & WFNS: $100 \%$ & WFNS: $100 \%$ & $6.0(0)$ & $6.0(0)$ \\
\hline Frazer et al., 2007 & $\begin{array}{l}\text { ACoA, ICA, MCA, } \\
\text { PCoA }\end{array}$ & $\begin{array}{c}\text { ACoA, BA, ICA, } \\
\text { MCA, PCoA }\end{array}$ & WFNS: $91 \%$ & WFNS: $100 \%$ & $\begin{array}{c}\text { 1st: } 0.4(0.3), 2 \text { nd: } \\
\quad 6.3(0.9)\end{array}$ & $\begin{array}{c}\text { 1st: } 0.4(0.3), 2 \mathrm{nd}: \\
6.1(1.3)\end{array}$ \\
\hline $\begin{array}{l}\text { Hadjivassiliou et al., } \\
2001\end{array}$ & $\begin{array}{l}\text { ACoA, ICA, MCA, } \\
\text { PCoA }\end{array}$ & $\begin{array}{l}\text { ACOA, ICA, MCA, } \\
\text { PCoA }\end{array}$ & WFNS: $78 \%$ & WFNS: $78 \%$ & $12.0(0)$ & $12.0(0)$ \\
\hline $\begin{array}{l}\text { Koivisto et al., } \\
2000 \ddagger\end{array}$ & $\begin{array}{l}\text { ACA, ICA, MCA, } \\
\text { PC }\end{array}$ & $\begin{array}{l}\text { ACA, ICA, MCA, } \\
\quad \text { PC }\end{array}$ & $\begin{array}{c}\text { GOS: 1st: 35\%, 2nd: } \\
53 \% \text {, 3rd: } 65 \%\end{array}$ & $\begin{array}{c}\text { GOS: 1st: 48\%, 2nd: } \\
63 \%, 3 \mathrm{rd}: 73 \%\end{array}$ & $\begin{array}{c}\text { 1st: } 0.3(0), 2 \text { nd: } 3.0 \\
\text { (0), 3rd: } 12.0(0)\end{array}$ & $\begin{array}{c}\text { 1st: } 0.3 \text { (0), 2nd: } 3.0 \\
\text { (0), 3rd: } 12.0(0)\end{array}$ \\
\hline $\begin{array}{l}\text { Ladowski et al., } \\
2014\end{array}$ & $\begin{array}{l}\text { ACoA, others } \\
\text { unspecified }\end{array}$ & $\begin{array}{l}\text { ACoA, others } \\
\text { unspecified }\end{array}$ & NA & NA & $20.9(22.3)$ & $20.9(22.3)$ \\
\hline Latimer et al., 2013 & $\begin{array}{l}\text { ACoA, ICA, MCA, } \\
\text { PCoA }\end{array}$ & $\begin{array}{l}\text { ACoA, ICA, MCA, } \\
\text { PCoA }\end{array}$ & $\mathrm{HH}: 56 \%$ & $\mathrm{HH}: 86 \%$ & $39.7(17.3)$ & $44.6(15.6)$ \\
\hline Preiss et al., 2007 & $\begin{array}{r}\text { ACoA, ICA, MCA, } \\
\text { OA, PC, PCoA }\end{array}$ & $\begin{array}{r}\mathrm{ACoA}, \mathrm{ICA}, \mathrm{MCA} \\
\mathrm{OA}, \mathrm{PC}, \mathrm{PCOA}\end{array}$ & $\mathrm{HH}: 2.0(0.96) \dagger$ & $\mathrm{HH}: 1.68(1.03) \dagger$ & $12.0(0)$ & $12.0(0)$ \\
\hline Proust et al., 2009 & $\mathrm{ACOA}$ & $\mathrm{ACoA}$ & $\mathrm{HH}: 64 \%$ & $\mathrm{HH}: 57 \%$ & $22(10.7)$ & $22(10.7)$ \\
\hline $\begin{array}{l}\text { Santiago-Ramajo } \\
\text { et al., } 2007\end{array}$ & $\begin{array}{l}\text { ACoA, MCA, } \\
\text { PCoA, others } \\
\text { unspecified }\end{array}$ & $\begin{array}{l}\text { ACoA, MCA, } \\
\text { PCoA, others } \\
\text { unspecified }\end{array}$ & $\begin{array}{l}\mathrm{HH}: 65 \% \text {, WFNS: } \\
\quad 73 \%\end{array}$ & $\begin{array}{l}\text { HH: } 79 \% \text {, WFNS: } \\
\quad 83 \%\end{array}$ & $4.1(0.6)$ & $4.3(0.6)$ \\
\hline Vieira et al., 2012 & $\begin{array}{c}\text { ACoA, ICA, MCA, } \\
\text { OA, PA, PCoA }\end{array}$ & $\begin{array}{c}\text { ACoA, ICA, MCA, } \\
\text { OA, PA, PCOA }\end{array}$ & $\mathrm{HH}: 100 \%$ & $\mathrm{HH}: 100 \%$ & $0.5(0.4)$ & $0.4(0.2)$ \\
\hline
\end{tabular}

$\mathrm{ACA}=$ anterior cerebral artery; $\mathrm{BA}=$ basilar artery; $\mathrm{GOS}=$ Glasgow outcome scale; $\mathrm{ICA}=$ internal carotid artery; $\mathrm{OA}=$ ophthalmic artery; $\mathrm{PA}=$ pericallosal artery; $\mathrm{PC}=$ posterior circulation; $\mathrm{PCOA}=$ posterior communicating artery; $\mathrm{PICA}=$ posterior inferior cerebellar artery.

* HH Grades I-II, WFNS Grades I-II, and GOS Grades 13-15 were considered good clinical outcomes.

$\dagger$ Mean (SD) HH grade, rather than the percentage of patients with good clinical grades, was provided.

$\ddagger$ Three testing sessions were included in this study with increasingly larger samples and consequently different sample characteristics: the 1 st session at discharge,

2nd session 3 months posttreatment, and a 3rd session at 12 months posttreatment.

size and location, although cognitive functioning is rarely taken into account. In this review we quantitatively summarized the literature comparing neuropsychological functioning after endovascular coiling and surgical clipping of SAH.

Our hypothesis that coiled patients would outperform clipped patients was only partially supported; neuropsy- chological test performance did not differ between treatment approaches for the most part, with differences centering on executive function and language tests. It is possible that because executive function and language tests engage complex, higher-order cognitive functions, they are more sensitive to the damage incurred by aneurysm rupture and perhaps during treatment. As the coiling pro-
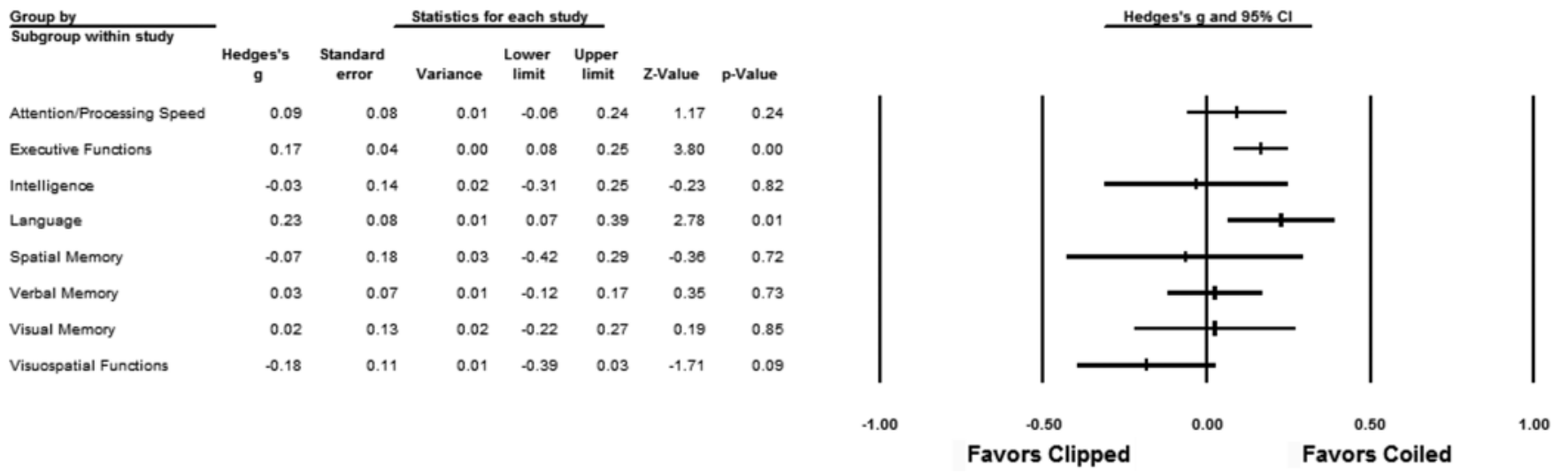

FIG. 2. Forest plot of Hedge's g $(95 \% \mathrm{Cl})$, comparing neuropsychological test performance between coiled and clipped patients. 


\begin{tabular}{|c|c|c|c|c|c|c|c|}
\hline \multirow{2}{*}{$\frac{\text { Group by }}{\text { Subgroup within study }}$} & \multicolumn{7}{|c|}{ Statistics for each study } \\
\hline & $\begin{array}{l}\text { Hedges's } \\
\mathbf{g}\end{array}$ & $\begin{array}{c}\text { Standard } \\
\text { error }\end{array}$ & Variance & $\begin{array}{l}\text { Lower } \\
\text { limit }\end{array}$ & $\begin{array}{l}\text { Upper } \\
\text { limit }\end{array}$ & z-Value & p-Value \\
\hline Attention/Processing Speed & -0.60 & 0.14 & 0.02 & -0.87 & -0.33 & -4.39 & 0.00 \\
\hline Executive Functions & -0.74 & 0.06 & 0.00 & -0.86 & -0.62 & -12.00 & 0.00 \\
\hline Intelligence & -0.93 & 0.21 & 0.04 & -1.33 & -0.53 & -4.52 & 0.00 \\
\hline Language & -0.52 & 0.21 & 0.04 & -0.92 & -0.12 & -2.54 & 0.01 \\
\hline Spatial Memory & -0.23 & 0.19 & 0.04 & -0.61 & 0.14 & -1.23 & 0.22 \\
\hline Verbal Memory & -0.53 & 0.20 & 0.04 & -0.92 & -0.14 & -2.67 & 0.01 \\
\hline Visual Memory & -0.85 & 0.18 & 0.03 & -1.20 & -0.51 & -4.86 & 0.00 \\
\hline Visuospatial Functions & -0.05 & 0.42 & 0.17 & -0.87 & 0.76 & -0.12 & 0.90 \\
\hline
\end{tabular}

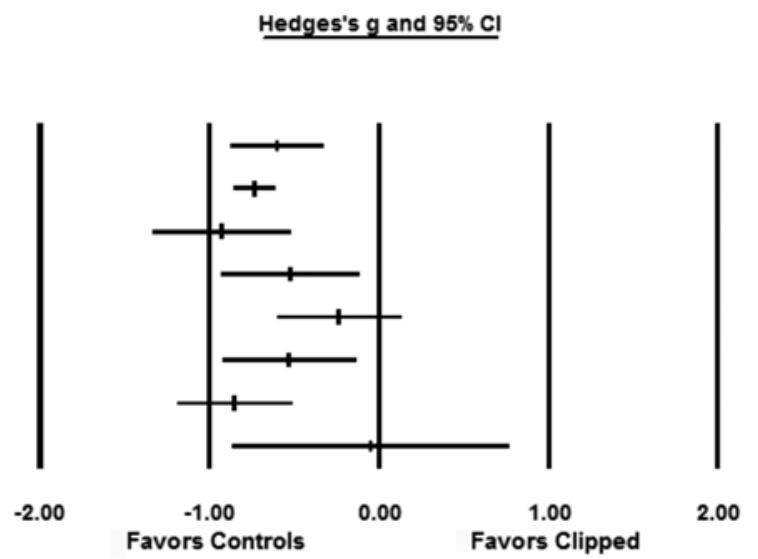

FIG. 3. Forest plot of Hedge's g $(95 \% \mathrm{Cl})$, comparing neuropsychological test performance between clipped patients and healthy controls.

cedure is less invasive than clipping, the recovery period tends to be shorter and can also influence test performance. Another factor to consider is the heterogeneity of executive function tests; given the relatively small number of studies available for inclusion in our study, subdomains within executive function could not be formed. Therefore, the multiple types of tests (for example, inhibition, working memory, set shifting) in the executive function domain remained, even though performance on these tests can be affected to varying degrees.

Two studies ${ }^{12,16}$ provided some information on the time course of cognitive changes; both tested patients approximately 10 days after treatment and conducted subsequent assessments at 6 months ${ }^{12}$ and at 3 and 12 months ${ }^{16}$ later. Both patient groups demonstrated minor improvement in a few domains in the months following treatment (although Koivisto and colleagues ${ }^{16}$ did not report the statistics). When comparing treatment groups on the medium-length follow-up sessions ( $3^{16}$ and $6^{12}$ months), clipped patients outperformed the coiled patients on the executive function and visuospatial domains, and almost significantly so on the intelligence domain. No group differences emerged at the 12-month follow-up. It is possible that clipping is associated with favorable neuropsychological performance in the early stages of recovery (that is, within 6 months posttreatment). This difference may eventually disappear and subsequently switch to a favorable performance in the coiled patients after a year (the majority of studies tested 12 months or longer posttreatment and found better performance in coiled vs clipped patients). This proposition is supported in part by the meta-regression for the testing delay (see below). However, it is important to note that the surgical patients in Frazer et al. ${ }^{12}$ had greater premorbid intelligence scores, as estimated by the National Adult Reading Test (clipped 103.45 vs coiled 90.33). Therefore, the unique findings of Frazer and colleagues may have been influenced by the preexisting group differences in intelligence that could skew their data at follow-up testing.

Beyond some unique neuropsychological differences between treatment approaches, the 2 patient groups were impaired on a wide range of cognitive tests relative to controls, as predicted. Deficits were most pronounced on visual memory for both groups and on intelligence measures for clipped patients. Medium to large effect sizes emerged on all domains except spatial memory and visuospatial functions. In general, clipped patients performed worse than coiled patients, in comparison with controls. The largest differences were in the executive function $(\mathrm{g}=$

\begin{tabular}{|c|c|c|c|c|c|c|c|}
\hline \multirow{2}{*}{$\frac{\text { Group by }}{\text { Subgroup within study }}$} & \multicolumn{7}{|c|}{ Statistics for each study } \\
\hline & $\underset{\mathrm{g}}{\text { Hedges's }}$ & $\begin{array}{l}\text { Standard } \\
\text { error }\end{array}$ & Variance & $\begin{array}{l}\text { Lower } \\
\text { limit }\end{array}$ & $\begin{array}{l}\text { Upper } \\
\text { limit }\end{array}$ & Z-Value & Value \\
\hline Attention/Processing Speed & -0.29 & 0.12 & 0.02 & -0.53 & -0.05 & -2.38 & 0.02 \\
\hline Executive Functions & -0.49 & 0.06 & 0.00 & -0.61 & -0.38 & -8.15 & 0.00 \\
\hline Intelligence & -0.43 & 0.20 & 0.04 & -0.81 & -0.04 & -2.16 & 0.03 \\
\hline Language & -0.40 & 0.16 & 0.03 & -0.71 & -0.09 & -2.51 & 0.01 \\
\hline Spatial Memory & -0.34 & 0.19 & 0.04 & -0.72 & 0.04 & -1.76 & 0.08 \\
\hline Verbal Memory & -0.55 & 0.17 & 0.03 & -0.88 & -0.23 & -3.30 & 0.00 \\
\hline Visual Memory & -0.81 & 0.18 & 0.03 & -1.15 & -0.46 & -4.59 & 0.00 \\
\hline Visuospatial Functions & 0.02 & 0.44 & 0.19 & -0.84 & 0.88 & 0.04 & 0.96 \\
\hline
\end{tabular}

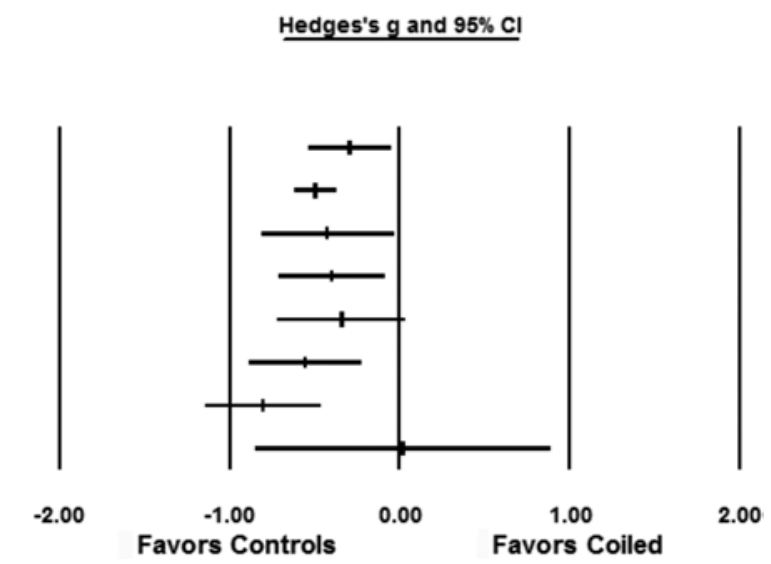

FIG. 4. Forest plot of Hedge's $\mathrm{g}(95 \% \mathrm{Cl})$, comparing neuropsychological test performance between coiled patients and healthy controls. 
-0.74 clipped vs -0.49 coiled) and intelligence domains (g $=-0.93$ clipped vs -0.43 coiled). Thus, treatment does not appear to return neuropsychological functioning to preexisting levels among SAH survivors. However, it is difficult to substantiate this claim because pretreatment data were only provided by Vieira and colleagues..$^{29}$ In that study, patients were tested 1-2 weeks after aneurysm rupture and again approximately 2 weeks after treatment. Though the neuropsychological functioning of coiled patients did not change with treatment, the performance of clipped patients deteriorated on multiple language and memory measures.

The results of the meta-regressions were mostly congruent with the hypotheses. Effect sizes increased modestly over the span of approximately 15 years during which the included studies were published. In other words, coiled patients scored better on neuropsychological tests in more recent years. This trend can probably be attributed, in part, to improvements in the endovascular method. Since coiling is relatively new, it is not surprising to see an improvement in functional outcome as a consequence of advances in the procedure. In contrast, clipping has been performed since the late 1930s, and the rate of advances in this procedure has slowed. Of note, the study publication year accounted for only $9 \%$ of the variance in group performance differences, suggesting that the influence of this variable is small.

Surprisingly, the testing delay was weakly (though still significantly) predictive of effect size, accounting for only $4 \%$ of the variance. The difference between groups (favoring coiled patients) increased as the interval between treatment and testing grew, a finding that could be interpreted in multiple ways. First, coiled patients may improve at a faster rate than clipped patients-at least in terms of neuropsychological functioning. Second, the weak relationship could simply be attributed to a ceiling effect; most studies tested patients more than a year after treatment, at which point patients may have reached or approached maximum recovery. Consequently, group differences may have been minimized. With that said, patients in either treatment group still performed worse than healthy controls, even in studies with the longest testing delays (approximately $21-28$ months posttreatment). ${ }^{3,17}$

Mean patient age emerged as a strong predictor of effect sizes, accounting for $48 \%$ of the variance. It appears that functional outcomes-from a neuropsychological perspective-appear to favor endovascular treatment in older patients. As with the above argument, it is possible that the less invasive nature of coiling is less taxing and more conducive to recovery.

Congruent with the hypothesis, higher rates of MCA ruptures were, in some domains, associated with smaller group differences. In particular, smaller effect sizes emerged in the language and executive function domains from studies with higher MCA rupture rates, indicative of a more similar performance between clipped and coiled groups. Given the angioanatomical features of MCA aneurysms (for example, small dome/neck ratios and arterial branches) that can prove more difficult for endovascular treatment, this finding is not surprising. Interestingly, the opposite pattern emerged on intelligence measures, with effect sizes increasing with MCA ruptures. A possible ex- planation for this finding is that because intelligence indices are amalgamations of a variety of cognitive processes, deficits in certain functions may be rescued by equal or superior performance from other functions that were unaffected. The predictions regarding posterior circulation rupture rates were not confirmed; effect sizes across the amalgamated domains, and specifically within the intelligence domain, decreased as posterior rupture rates increased (indicating smaller differences in group performance). This finding must be interpreted with caution. The included studies had low rates of posterior circulation ruptures, ranging from $0 \%$ to $11 \%$, and this limited range may have hindered the predictive power of the regression model. Anterior communicating artery rupture rates were not predictive of effect sizes in any domain. This finding was consistent with the hypothesis given the lack of angioanatomical contraindications for either clipping or coiling ACoA ruptures.

\section{Study Limitations}

A caveat to our findings is the typical clinical grade distributions of patients upon recruitment into the studies. A majority of patients in almost all studies had a good neurological status on admission, as indicated by a WFNS or Hunt and Hess (HH) Grade I or II. The one exception was the group of patients described by Proust and colleagues, who had a higher proportion of HH Grade III-IV cases (36\% clipped and $43 \%$ coiled). ${ }^{23}$ Undoubtedly, a major reason for excluding patients with worse clinical grades is a logistical one, as individuals with poor functional capabilities may be unable or unwilling to complete a demanding neuropsychological battery. Nevertheless, the results are biased by the overrepresentation of "ideal" SAH patients; test performance differences may be limited when primarily cases with good functional presentations are compared, although fewer such cases are available after neurosurgery.

A caveat specific to the rupture location analysis is the degree of group homogeneity. Studies with higher rates of a certain rupture location may have a sample that is more similar and, consequently, also has smaller group variability. This can increase the likelihood of detecting group differences.

Several general limitations exist in the field, a major one being the lack of premorbid assessments. This is not currently feasible in patients with unruptured intracranial aneurysms since the rupture rate is very low. In addition, most studies in the literature (and none of the included studies) do not randomize treatment methods for patients - the International Subarachnoid Aneurysm Trial being one of the few exceptions. Within the realm of stroke treatment, much of the nonrandomization can be attributed to angioanatomical contraindications to either coiling or clipping. However, nonrandom allocation may give rise to group differences that are difficult to control for-either through study design or statistically so-at both the individual study and meta-analysis levels. Finally, because the size of aneurysms can also influence the choice of treatment, ${ }^{15}$ this factor would have been pertinent to our analyses. Unfortunately, not enough studies provided sufficient details to include aneurysm size as a variable. 


\section{Conclusions}

Although the literature on neuropsychological functioning after SAH treatment is relatively sparse, some consistent trends are apparent. Performance on executive function and language measures tends to be better after coiling than after clipping. Just as coiling procedures improved over the years, so did effect sizes, suggesting that the greater efficacy is also apparent in improved functional outcomes. Regardless of the treatment modality, all patients performed worse than healthy controls, and the neuropsychological impairments appear to be maintained over time. Moreover, clipped patients outperformed coiled patients, a difference that disappeared and switched to a favorable performance with coiling after a year; however, this finding should be interpreted with caution given the small analysis and unique sample characteristics. Patient age was a significant predictor of effect size, indicating that coiling may be a better alternative for older patients, at least in terms of neuropsychological outcomes. Lastly, rupture location may have some effect on test performance. Thus, although the neuropsychological impairments of clipped patients were not universally observed, they occurred frequently enough to warrant consideration, in conjunction with the generally favorable functional outcomes of endovascular coiling (compare with $\mathrm{Li}$ et al. ${ }^{19}$ ), when deciding on treatment approaches.

Elucidation would further inform the care of patients with unruptured intracranial aneurysms, as knowledge of the potential neuropsychological impacts of rupture (in addition to other variables related to the risk of rupture itself) could affect the decision to provide prophylactic treatment to obviate SAH and its sequelae. Lastly, additional studies of neuropsychological performance in SAH patients, especially the time course of these deficits, could better inform posttreatment rehabilitation approaches to remedy neuropsychological deficits and, ultimately, to improve quality of life for these patients.

\section{Acknowledgments}

Mr. Egeto was supported by a Canada Graduate Scholarships Master's Award, Canadian Institutes of Health Research, and Dr. Schweizer was supported by an Early Researcher Award from the Ontario Ministry of Research and Innovation.

\section{References}

1. Akyuz M, Erylmaz M, Ozdemir C, Goksu E, Ucar T, Tuncer $\mathrm{R}$ : Effect of temporary clipping on frontal lobe functions in patients with ruptured aneurysm of the anterior communicating artery. Acta Neurol Scand 112:293-297, 2005

2. Al-Khindi T, Macdonald RL, Schweizer TA: Cognitive and functional outcome after aneurysmal subarachnoid hemorrhage. Stroke 41:e519-e536, 2010

3. Bellebaum C, Schäfers L, Schoch B, Wanke I, Stolke D, Forsting M, et al: Clipping versus coiling: neuropsychological follow up after aneurysmal subarachnoid haemorrhage (SAH). J Clin Exp Neuropsychol 26:1081-1092, 2004

4. Borenstein M, Hedges L, Higgins J, Rothstein H: Comprehensive Meta-Analysis Version 2. Engelwood, NJ: Biostat, 2005 (https://www.meta-analysis.com/downloads/MetaAnalysis-Manual.pdf) [Accessed January 18, 2017]

5. Brinjikji W, Lanzino G, Rabinstein AA, Kallmes DF, Cloft,
HJ: Age-related trends in the treatment and outcomes of ruptured cerebral aneurysms: a study of the nationwide inpatient sample 2001-2009. AJNR Am J Neuroradiol 34:10221027,2013

6. Chan A, Ho S, Poon WS: Neuropsychological sequelae of patients treated with microsurgical clipping or endovascular embolization for anterior communicating artery aneurysm. Eur Neurol 47:37-44, 2002

7. Duval S, Tweedie R: Trim and fill: A simple funnel-plotbased method of testing and adjusting for publication bias in meta-analysis. Biometrics 56:455-463, 2000

8. Egger M, Davey Smith G, Schneider M, Minder C: Bias in meta-analysis detected by a simple, graphical test. BMJ 315:629-634, 1997

9. Escartin G, Junqué C, Juncadella M, Gabarrós A, de Miquel MA, Rubio F: Decision-making impairment on the Iowa Gambling Task after endovascular coiling or neurosurgical clipping for ruptured anterior communicating artery aneurysm. Neuropsychology 26:172-180, 2012

10. Feigin VL, Lawes CM, Bennett DA, Barker-Collo SL, Parag V: Worldwide stroke incidence and early case fatality reported in 56 population-based studies: a systematic review. Lancet Neurol 8:355-369, 2009

11. Fontanella M, Perozzo P, Ursone R, Garbossa D, Bergui M: Neuropsychological assessment after microsurgical clipping or endovascular treatment for anterior communicating artery aneurysm. Acta Neurochir (Wien) 145:867-872, 2003

12. Frazer D, Ahuja A, Watkins L, Cipolotti L: Coiling versus clipping for the treatment of aneurysmal subarachnoid hemorrhage: a longitudinal investigation into cognitive outcome. Neurosurgery 60:434-442, 2007

13. Hadjivassiliou M, Tooth CL, Romanowski CA, Byrne J, Battersby RD, Oxbury S, et al: Aneurysmal SAH: cognitive outcome and structural damage after clipping or coiling. Neurology 56:1672-1677, 2001

14. Jaja BNR, Lingsma H, Steyerberg EW, Schweizer TA, Thorpe KE, Macdonald RL: Neuroimaging characteristics of ruptured aneurysm as predictors of outcome after aneurysmal subarachnoid hemorrhage: pooled analyses of the SAHIT cohort. J Neurosurg 124:1703-1711, 2016

15. Johnston SC, Higashida RT, Barrow DL, Caplan LR, Dion JE, Hademenos G, et al: Recommendations for the endovascular treatment of intracranial aneurysms: a statement for healthcare professionals from the Committee on Cerebrovascular Imaging of the American Heart Association Council on Cardiovascular Radiology. Stroke 33:2536-2544, 2002

16. Koivisto T, Vanninen R, Hurskainen H, Saari T, Hernesniemi J, Vapalahti M: Outcomes of early endovascular versus surgical treatment of ruptured cerebral aneurysms. A prospective randomized study. Stroke 31:2369-2377, 2000

17. Ladowski D, Qian W, Kapadia AN, Macdonald RL, Schweizer TA: Effect of aneurysmal subarachnoid hemorrhage on word generation. Behav Neurol 2014:610868, 2014

18. Latimer SF, Wilson FC, McCusker CG, Caldwell SB, Rennie I: Subarachnoid haemorrhage (SAH): long-term cognitive outcome in patients treated with surgical clipping or endovascular coiling. Disabil Rehabil 35:845-850, 2013

19. Li H, Pan R, Wang H, Rong X, Yin Z, Milgrom DP, et al: Clipping versus coiling for ruptured intracranial aneurysms: a systematic review and meta-analysis. Stroke 44:29-37, 2013

20. Moher D, Liberati A, Tetzlaff J, Altman DG: Preferred reporting items for systematic reviews and meta-analyses: the PRISMA statement. J Clin Epidemiol 62:1006-1012, 2009

21. Molyneux AJ, Kerr RS, Yu LM, Clarke M, Sneade M, Yarnold JA, et al: International subarachnoid aneurysm trial (ISAT) of neurosurgical clipping versus endovascular coiling in 2143 patients with ruptured intracranial aneurysms: a randomised comparison of effects on survival, dependency, 
seizures, rebleeding, subgroups, and aneurysm occlusion. Lancet 366:809-817, 2005

22. Preiss M, Koblihova J, Netuka D, Klose J, Charvat F, Benes V: Ruptured cerebral aneurysm patients treated by clipping or coiling: comparison of long-term neuropsychological and personality outcomes. Zentralbl Neurochir 68:169-175, 2007

23. Proust F, Martinaud O, Gérardin E, Derrey S, Levèque S, Bioux S, et al: Quality of life and brain damage after microsurgical clip occlusion or endovascular coil embolization for ruptured anterior communicating artery aneurysms: neuropsychological assessment. J Neurosurg 110:19-29, 2009

24. Regli L, Uske A, de Tribolet N: Endovascular coil placement compared with surgical clipping for the treatment of unruptured middle cerebral artery aneurysms: a consecutive series. J Neurosurg 90:1025-1030, 1999

25. Santiago-Ramajo S, Katati MJ, Pérez-García M, Coín-Mejias MA, Vilar-Lopez R, Caracuel-Romero A, et al: Neuropsychological evaluation of the treatments applied to intracranial aneurysms in a Spanish sample. J Clin Exp Neuropsychol 29:634-641, 2007

26. Stienen MN, Weisshaupt R, Fandino J, Hildebrandt G, Studerus-Germann A, Schatlo B: Characteristics of patients without neuropsychological deficits following aneurysmal subarachnoid haemorrhage. Acta Neurochir Suppl 120:125129,2015

27. Tam AKH, Kapadia A, Ilodigwe D, Li Z, Schweizer TA, Macdonald RL: Impact of global cerebral atrophy on clinical outcome after subarachnoid hemorrhage. J Neurosurg 119:198-206, 2013

28. Taylor TN, Davis PH, Torner JC, Holmes J, Meyer JW, Jacobson MF: Lifetime cost of stroke in the United States. Stroke 27:1459-1466, 1996

29. Vieira ACC, Azevedo-Filho HRC, Andrade G, Costa e Silva IE, de Fátima Leal Griz M, Quinino S, et al: Cognitive changes in patients with aneurysmal subarachnoid hemorrhage before and early posttreatment: differences between surgical and endovascular. World Neurosurg 78:95-100, 2012

30. Vieira ACC, Azevedo-Filho HRC, Quinino S, Ponte de Souza ML, Câmara D Jr, Leitão L, et al: Language, memory, and verbal fluency changes in patients with aneurysmal subarachnoid hemorrhage: results of a preoperative investigation. World Neurosurg 75:653-659, 596-597, 2011

31. Wells G, Shea B, O'Connell D, Peterson J, Welch V, Losos $M$, et al: The Newcastle-Ottawa scale (NOS) for assessing the quality of nonrandomised studies in meta-analyses. Ottawa Hospital.(http://www.ohri.ca/programs/clinical_epidemiology/ oxford.asp) [Accessed January 18, 2017]

32. Wong GKC, Lam SW, Wong A, Mok V, Siu D, Ngai K, et al: Early MoCA-assessed cognitive impairment after aneurysmal subarachnoid hemorrhage and relationship to 1-year functional outcome. Transl Stroke Res 5:286-291, 2014

\section{Disclosures}

Dr. Macdonald has ownership of and owns stock in Edge Therapeutics Inc. and has received support from the Brain Aneurysm Foundation, Physicians Services Inc. Foundation, Heart and Stroke Foundation of Canada, and Canadian Institutes of Health Research for non-study-related clinical or research effort.

\section{Author Contributions}

Conception and design: Egeto. Acquisition of data: Egeto. Analysis and interpretation of data: Egeto. Drafting the article: Egeto. Critically revising the article: Schweizer, Macdonald, Ornstein. Reviewed submitted version of manuscript: Egeto. Statistical analysis: Egeto. Study supervision: Schweizer, Macdonald, Ornstein.

\section{Correspondence}

Tom A. Schweizer, St. Michael's Hospital, Neuroscience Research, 30 Bond St., Toronto, ON M5B 1W8, Canada. email: schweizert@smh.toronto.on.ca. 